\title{
Femtosecond Lorentz Microscopy for the Mapping of Ultrafast Magnetization Dynamics
}

Nara Rubiano da Silva ${ }^{1}$, Marcel Möller ${ }^{1}$, Armin Feist ${ }^{1}$, Henning Ulrichs ${ }^{2}$, Claus Ropers ${ }^{1}$ and Sascha Schäfer ${ }^{1,3 *}$

1. University of Göttingen, IV. Physical Institute, Göttingen 37077, Germany

2. University of Göttingen, I. Physical Institute, Göttingen 37077, Germany

3. University of Oldenburg, Institute of Physics, Oldenburg 26129, Germany

* Corresponding author: sascha.schaefer@uni-oldenburg.de

Time-resolved and in-situ electron microscopy techniques offer a unique access to dynamical processes at the nanoscale. Pushing the limits of temporal resolution, ultrafast transmission electron microscopy (UTEM) [1,2] utilizes ultrashort electron pulses to stroboscopically map transient states of matter. Recent advances in nanoscale photocathode technology allowed for the generation of high-coherence electron pulses with a temporal duration down to $200 \mathrm{fs}$, focusable to sub-nanometer spot-sizes and with a narrow spectral width of $0.6 \mathrm{eV}[2,3]$. High quality electron pulses now open the possibility to equip a broad range of electron microscopy techniques with femtosecond temporal resolution, including bright-field imaging [4], electron-energy spectroscopy [5] or convergent-beam electron diffraction [6].

Here, we report on femtosecond Lorentz microscopy for the imaging of ultrafast demagnetization dynamics in a single nanostructure [7], extending the accessible time-scales of time-resolved Lorentz microscopy [8-11]. In the experiment, an isolated permalloy disc (1- $\mu \mathrm{m}$ diameter, 20-nm thickness) on a silicon nitride membrane is optically excited by femtosecond laser pulses (800-nm central wavelength, $50-\mathrm{fs}$ pulse duration, $500-\mathrm{kHz}$ repetition rate). The magnetic texture of the disc is mapped by collimated electron pulses with a temporal duration of 700-fs using Lorentz microscopy (Fig. 1a). The disc hosts a magnetic vortex state, visible in the Lorentz micrographs as an image intensity peak at the center of the disc (Fig. 1b). Notably, Lorentz micrographs recorded in the stroboscopic UTEM mode (Fig. 1b, lower panel) show an image contrast comparable to micrographs recorded with a continuous Schottky electron source (Fig. 1b, upper panel), highlighting the spatial coherence of the photoelectron pulses.

Upon optical excitation, the spin system in the permalloy disc is heated close to the Curie temperature, resulting in a decreased sample magnetization. In the micrographs, the image intensity of the central vortex spot directly correlates with the sample magnetization. Radial profiles of the Lorentz micrographs for different delay times between optical excitation and electron probing are shown in Fig. 1c. A sudden decrease of the central peak is observed after excitation with a partial recovery on picosecond time-scales. Tracking the central peak amplitude with delay time gives a direct measure for the temporal evolution of the vortex magnetization at different optical fluences (Fig. 1d). The observed changes are in line with results from ultrafast optical spectroscopy [12]: In a microscopic picture, optical excitation couples to the electronic subsystem of the sample. Electron-spin coupling on few $100 \mathrm{fs}$, yields the transiently hot spin system observed here. The time scale of partial spin cooling (about 2 ps, Fig. 1d) is governed by electronlattice coupling.

Whereas femtosecond magnetization dynamics are studied in detail by ultrafast optical spectroscopy and time-resolved photoemission spectroscopy, femtosecond Lorentz microscopy ideally complements these investigations by adding real-space spatial information. In particular, in the present study, a spatial 
resolution below $100 \mathrm{~nm}$ is obtained and could by employed in future investigations for mapping the ultrafast spatially evolution of optically excited nanoscale magnetic textures.

\section{References:}

[1] DJ Flannigan and AH Zewail, Acc Chem Res 45 (2012), p. 1828.

[2] A Feist et al., Ultramicroscopy 176 (2017), p. 63.

[3] N Bach et al., Structural Dynamics 6 (2019), p. 014301.

[4] DR Cremons, DA Plemmons and DJ Flannigan, Nature Communications 7 (2016), p. 11230.

[5] A Feist et al., Nature 521 (2015), p. 200.

[6] A Feist et al., Struct Dyn 5 (2018), p. 014302.

[7] N Rubiano da Silva et al., Physical Review X 8 (2018), p. 031052.

[8] HS Park, JS Baskin and AH Zewail, Nano Lett 10 (2010), p. 3796.

[9] KB Schliep et al., Appl Phys Lett 110 (2017), p.

[10] G Berruto et al., Phys Rev Lett 120 (2018), p.

[11] T Eggebrecht et al., Phys Rev Lett 118 (2017), p. 097203.

[12] E Beaurepaire et al., Phys Rev Lett 76 (1996), p. 4250.

[13] The authors acknowledge funding by the Deutsche Forschungsgemeinschaft (DFG-SPP-1840 "Quantum Dynamics in Tailored Intense Fields," and DFG-SFB-1073 "Atomic Scale Control of Energy Conversion," projects A05 and A06), support by the Lower Saxony Ministry of Science and Culture and funding of the instrumentation by the DFG and VolkswagenStiftung. N. R. S. would like to acknowledge the support by the Conselho Nacional de Desenvolvimento Científico e Tecnológico (Science Without Borders Program, Governo Dilma Rousseff, Brazil).
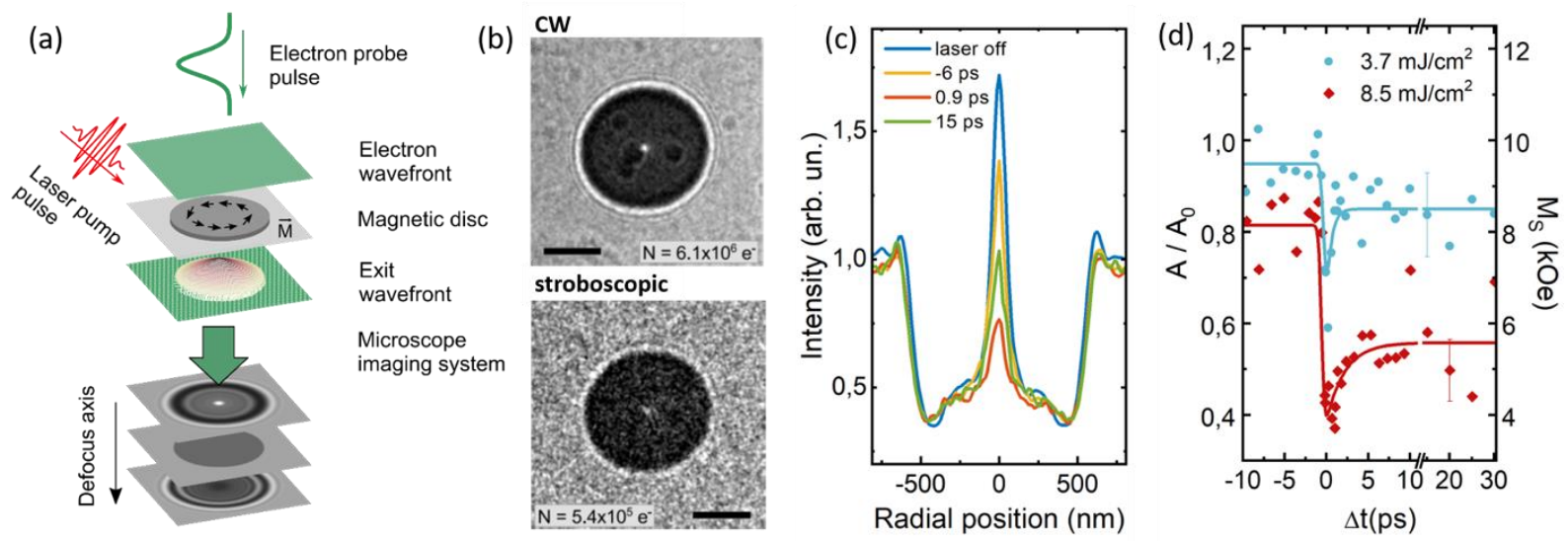

Figure 1. Femtosecond Lorentz microscopy. (a) Femtosecond electron pulses image the transient magnetic state in an optically excited nanostructure employing Lorentz microscopy. (b) Lorentz micrographs of a permalloy disc, recorded with a continuous electron beam (upper panel) and with a pulsed electron beam derived from a laser-driven tip-shaped photocathode (lower panel). N: number of imaging electrons; scale bar: $500 \mathrm{~nm}$ (c) Radial profiles of femtosecond Lorentz micrographs for different delay times between optical excitation and electron probing. Continuous heating of the high-repetition rate laser results in a difference in peak amplitude between negative delay times and for experiments with no laser on the sample. (d) Temporal evolution of the image intensity of the central peak in the Lorentz micrograph (panel c) for two different optical fluences. Quantitative analysis of Lorentz contrast recovers the transient sample magnetization (right axis). Adapted from Ref. [7]. 